\title{
Identification of tumorigenicity-associated genes in osteosarcoma cell lines based on bioinformatic analysis and experimental validation
}

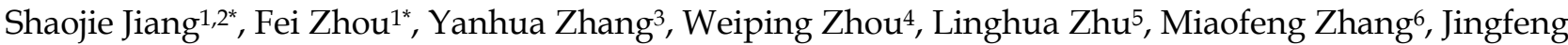

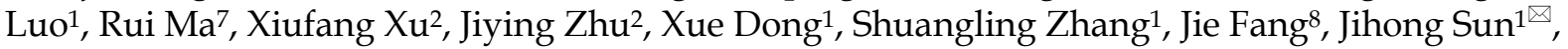 \\ Xiaoming Yang $1,9 \llbracket$ \\ 1. Department of Radiology, Sir Run Run Shaw Hospital, School of Medicine, Zhejiang University, Hangzhou, Zhejiang, 310016, China. \\ 2. School of Medical Imaging, Hangzhou Medical College, Hangzhou, Zhejiang, 310013, China. \\ 3. Department of Pathology, Sir Run Run Shaw Hospital, School of Medicine, Zhejiang University, Hangzhou, Zhejiang, 310016, China. \\ 4. Department of Diagnostic Ultrasound and Echocardiography, Sir Run Run Shaw Hospital, School of Medicine, Zhejiang University, Hangzhou, Zhejiang, \\ 310016, China. \\ 5. Department of General Surgery, Sir Run Run Shaw Hospital, School of Medicine, Zhejiang University, Hangzhou, Zhejiang, 310016, China. \\ 6. Department of Orthopedic Surgery, Second Affiliated Hospital, School of Medicine, Zhejiang University, Hangzhou, Zhejiang, 310009, China. \\ 7. Department of Surgery, Zhejiang University Hospital, Zhejiang University, Hangzhou, Zhejiang 310027, China \\ 8. Key Laboratory of Experimental Animal and Safety Research, Zhejiang Academy of Medical Sciences, Hangzhou, Zhejiang 310013, China. \\ 9. Image-Guided Bio-Molecular Intervention Research, Department of Radiology, University of Washington School of Medicine, Seattle, Washington, 98109, \\ USA.
}

*Shaojie Jiang and Fei Zhou contributed equally to this work.

$\triangle$ Corresponding authors: Xiaoming Yang, MD, PhD, Department of Radiology, Sir Run Run Shaw Hospital, School of Medicine, Zhejiang University, Hangzhou, Zhejiang, 310016, China. xmyang@zju.edu.cn; or Image-Guided Bio-Molecular Intervention Research, Department of Radiology, University of Washington School of Medicine, Seattle, Washington, 98109, USA. xmyang@uw.edu. Or Jihong Sun, MD, PhD, Department of Radiology, Sir Run Run Shaw Hospital, School of Medicine, Zhejiang University, Hangzhou, Zhejiang, 310016, China. sunjihong@zju.edu.cn.

(1) The author(s). This is an open access article distributed under the terms of the Creative Commons Attribution License (https://creativecommons.org/licenses/by/4.0/). See http:/ /ivyspring.com/terms for full terms and conditions.

Received: 2019.06.07; Accepted: 2020.03.03; Published: 2020.03.26

\begin{abstract}
Osteosarcoma is the most common primary malignant tumor of bone. Tumorigenic investigation of osteosarcoma cell lines may facilitate preclinical studies of targeted therapy. Therefore, the aim of this study was to explore the tumorigenicity-associated genes in osteosarcoma cells. We found that 138 genes were highly expressed and 86 genes were lowly expressed in highly tumorigenic osteosarcoma cell lines (143B, MNNG/HOS, and SJSA-1) compared with poorly tumorigenic osteosarcoma cell lines (MG-63, Saos-2, and U-2 OS). Kyoto Encyclopedia of Genes and Genomes (KEGG) analysis revealed that highly expressed genes were associated with amino acids and energy metabolism, while lowly expressed genes were associated with cell cycle and DNA replication. Gene Ontology (GO) analysis showed that highly expressed genes were associated with endoplasmic reticulum stress response and aggrephagy, whereas lowly expressed genes were correlated with extracellular matrix assembly and DNA damage response. Further analysis identified six highly expressed genes and six lowly expressed genes. Three of highly expressed genes (DDX10, FOXA2, and $H E Y I)$ were correlated with poor prognosis, while three of lowly expressed genes (CYP26BI, GPIBB, and IFI44) showed the opposite trend in patients with osteosarcoma. Knockdown of $H E Y I$ significantly inhibited the tumorigenicity of $143 B$ cells in BALB/c nude mice.
\end{abstract}

Key words: osteosarcoma, bioinformatic analysis, tumorigenicity, HEY1, prognosis

\section{Introduction}

Osteosarcoma is the most common primary malignant tumor of bone that most commonly affects children, adolescents, and young adults, with a global incidence of approximately one to three cases per 
million annually [1, 2]. Surgical resection was the primary treatment before 1970 . However, the survival of osteosarcoma patients treated with surgery alone is less than $20 \%$ [3]. The long-term survival rate was improved to $65-70 \%$ after the advent of multiagent chemotherapy regimens in the early 1970s, especially the combination of high-dose methotrexate, doxorubicin, and cisplatin (MAP) [4].

Osteosarcoma exhibits a predilection for the long bones near the metaphyseal growth plate and most commonly occurs in the distal femur (43\%), proximal tibia $(23 \%)$, or humerus $(10 \%)[1,2,5]$. The incidence is higher in adolescents of age 15-19, with eight to eleven cases per million annually, accounting for $>10 \%$ of all solid cancers [6]. The patients with localized osteosarcoma that respond to preoperative combination chemotherapy always show good prognosis [7]. However, the overall 5-year survival rate for patients with metastatic or relapsed osteosarcoma has remained at about $20 \%$ over the past 30 years [8]. The lung is the most common site of osteosarcoma metastasis (more than $85 \%$ of cases), with bone the second most common site [5]. Thus, seeking specific targets for early diagnosis and effective treatment is necessary.

Although candidate oncogenes (e.g., RUNX2, VEGFA, MDM2, and PRIM1) [9-12] and tumor suppressors (e.g., TP53, RB1, and PTEN) [13-15] have been identified in human osteosarcoma, the search for common molecular therapeutic targets in osteosarcoma has been disappointing to date [2]. Current clinical trials are focused on targeting the bone microenvironment (mainly osteoclasts and bone seeking) [8, 16], receptor tyrosine kinases (including Kit, VEGFR, FGFR, PDGFR, HER2, and IGF1R) [17-19], intracellular signaling pathways (SRC, mTOR, RAF, Aurora kinase A, and histone deacetylase) [20], and immune system [21-23]. Mifamurtide as an innate immune stimulant has shown clinical promise in the treatment of osteosarcoma [22, 23]. However, the strategies that target the bone microenvironment and signaling pathways remain controversial. For example, the Hedgehog, Notch, and WNT pathways have been implicated in both osteosarcoma and normal bone development, and targeted therapy may be problematic for children.

Tumorigenicity is defined as the ability of cells to give rise to tumors. Tumorigenicity of osteosarcoma cell lines in nude mouse is an important method for candidate target and drug screening. However, the mechanism remains obscure. Herein, the tumorigenic genes in osteosarcoma cell lines were identified by bioinformatic analysis, and Hes related family bHLH transcription factor with YRPW motif 1 (HEY1) was identified as a key factor in tumorigenicity of osteosarcoma cells by subsequent experimental validation.

\section{Methods}

\section{Cell lines}

Six osteosarcoma cell lines were obtained from American Type Culture Collection (ATCC) (https:// www.atcc.org/). SJSA-1 (also known as OSA, ATCC: CRL-2098) and U-2 OS (ATCC: HTB-96) were cultured in RPMI-1640 (Gibco, 11875-085) containing 10\% fetal bovine serum (FBS) (Gibco, 1099-141); 143B (ATCC: CRL-8303), MINNG/HOS Cl \#5 [R-1059-D] (ATCC: CRL-1547), and MG-63 (ATCC: CRL-1427) were cultured in minimum essential medium (MEM) (Gibco, 11095-080) containing 10\% FBS; Saos-2 (ATCC: HTB-85) was cultured in McCoy's 5A (Gibco, 16600-082) containing 15\% FBS. All cell lines were maintained at $37^{\circ} \mathrm{C}$ with $5 \% \mathrm{CO}_{2}$.

\section{In vivo tumorigenicity}

Osteosarcoma cell lines MG-63, Saos-2, U-2 OS, 143B, MNNG/HOS, and SJSA-1 were used for xenograft transplantation in $\mathrm{BALB} / \mathrm{C}$ nude mice according to a previous report [24]. Briefly, $1 \times 10^{6}$ cells in $100 \mu \mathrm{L}$ serum-free phosphate-buffered saline (PBS) were injected subcutaneously into each flank of locally bred BALB/c-nu mice (5 weeks old, female; five mice per group). The tumor size was measured every 5 days, and tumor volume was calculated by the formula (length $\times$ width $\times$ width) $/ 2$. For the highly tumorigenic cell lines, the experiments were stopped within a month. For the poorly tumorigenic cell lines, the experiments were stopped after 90 days.

\section{Quantitative polymerase chain reaction (qPCR) analysis}

Total cellular RNA was extracted from osteosarcoma cells or stable shRNA-expressing 143B cells $(80-90 \%$ confluence) using TRIzol reagent (Invitrogen, 15596026). The RNA concentration was measured by the NanoDrop 2000 (Thermo Fisher Scientific, ND2000). The first-strand cDNA was synthesized from $2 \mu \mathrm{g}$ of total RNA with random primers, and subsequent qPCR was performed using the GoTaq RT-qPCR kit (Promega, A6010). The experiment was carried out with the ViiA 7 Real-Time PCR System (Applied Biosystems, 4453534), and expression of mRNA was assessed based on the threshold cycle $(\mathrm{Ct})$. The relative mRNA expression levels were calculated as 2-[(Ct of mRNA)-(Ct of GAPDH)] after normalization to GAPDH expression. $\mathrm{qPCR}$ primer sequences $\left(F\right.$, forward; $R$, reverse; $\left.5^{\prime} \rightarrow 3^{\prime}\right)$ were as follows: CYP26B1-F: GTTCTGCCTCGGAGCTGATT, CYP26B1-R: CAACCCAATCCCCCTGACAA; GP1BB 
-F: AGACCACGTGGGACAGAACT, GP1BB-R: CAG GGTCTGGACCGCATTG; IFI44-F: TGGGAGCTGGA CCCTGTAAA, IFI44-R: CCTCCCTTAGATTCCCTAT TTGCT; DDX10-F: ATGTACTTGGAGCGGCCAAA, DDX10-R: CCCAGCCCATCTGTTGAAGT; FOXA2-F: CTTCAAGTGCGAGAAGCAGC, FOXA2-R: CCGAG TTGAGCCTGTGAGG; HEY1-F: AGTTAGGAGAGA GCCGCTGA，HEY1-R: TGTTGCTGGGGCTGGTAA AT; GAPDH-F: AGGTCGGAGTCAACGGATTT; GAPDH-R: ATGAAGGGGTCATTGATGGCA.

\section{Virus production and transfection}

shRNAs targeting HEY1 were cloned into the pLent-U6-RFP-Puro (Vigne, \#LT88024) vector. Targeting sequences were as follows: shRNA-Ctr: 5'-GCACCCAGUCCGCCCUGAGCAAA-3'; shHEY11: 5'-GCAGGAGGGAAAGGUUACUUU-3'; shHEY12: 5'-CCCAACUACAUCUUCCCAGAU-3'. The 293T cell line was used for lentivirus packaging. Briefly, a $10-\mathrm{cm}$ dish of $4 \times 10^{6}$ non-confluent $293 \mathrm{~T}$ cells were co-transfected with recombinant pLent-U6-RFP-Puro (Vigene, LT88024), pMD2G (Addgene, \#12259), and psPAX2 (Addgene, \#12260) for knock-down (RNAi), and pLent-C-HA (Vigene, LT88007), pMD2G, and psPAX2 for overexpression. The lentivirus-containing supernatant was harvested after $48 \mathrm{~h}$ and used for the subsequent experiment.

\section{Western blot analysis}

All cell lines were harvested at 80-90\% confluence and then lysed in radioimmunoprecipitation assay (RIPA) buffer (Thermo Fisher Scientific, \#89901). Protein concentration was measured with a bicinchoninic acid (BCA) protein assay kit (Thermo Fisher Scientific, \#23225). Protein samples were resolved by $8-10 \%$ sodium dodecyl sulfatepolyacrylamide gel electrophoresis (SDS-PAGE) and transferred to a polyvinylidene difluoride (PVDF) membrane (Bio-Rad, \#162-0117). The membrane was blocked in Tris-buffered saline (TBS) containing $0.05 \%$ Tween-20 (Amresco, 0777-1L) with 5\% nonfat skim milk (BD, \#232100) for $1 \mathrm{~h}$ at room temperature, followed by overnight incubation with primary antibodies at $4^{\circ} \mathrm{C}$. After three washes in TBS with Tween-20 (TBST), the membrane was incubated with horseradish peroxidase (HRP)-conjugated secondary antibody for $1 \mathrm{~h}$ at room temperature. After three washes in TBST, the membrane was treated with the EZ-ECL kit (Biological Industries, \#20-500-120) and visualized using the Tanon-5200 multi-automatic chemiluminescence/fluorescence imaging analysis system (Tanon Science \& Technology Inc). The following antibodies were used: anti-HEY1 (Abcam, ab154077), anti- $\beta$-actin (CST, \#3700), and secondary antibodies including anti-rabbit IgG, HRP-linked antibody (CST, \#7074) and anti-mouse IgG, HRP-linked antibody (CST, \#7076).

\section{In vivo fluorescence imaging}

Images of 143B cell-bearing mice were acquired using the Clairvivo OPT plus imaging system (SHIMADZU). Briefly, mice were anesthetized by $2 \%$ isoflurane gas for $5 \mathrm{~min}$ using the RC2 Anesthesia Machine (VetEquip). Then, postanesthetic mice were imaged under $530-\mathrm{nm}$ laser irradiation at the experimental endpoint.

\section{Microarray datasets}

The microarray datasets in this study were obtained from the Gene Expression Omnibus (GEO, http://www.ncbi.nlm.nih.gov/geo/). Gene expression profiles (GSE36001 and GSE42352) were provided by Professor Leonardo A. Meza-Zepeda [25] and Professor Anne-Marie Cleton-Jansen [26], respectively. Both GSE36001 and GSE42352 contain the gene expression profiles of osteosarcoma cell lines MG-63, Saos-2, U-2 OS, 143B, MNNG/HOS, and SJSA-1 (or OSA). The Sanger software package (V1.0.8) (https://shengxin.ren/softs/Sanger_V1.0.8. zip) was used for volcano plot calculation and visualization.

\section{Signaling pathway, biological process, and protein-protein interaction (PPI) network enrichment analyses}

For signaling pathway and biological process enrichment analyses, the high-expression group containing 138 genes and low-expression group containing 86 genes in highly tumorigenic osteosarcoma cell lines (143B, MNNG/HOS, and SJSA-1) as compared with poorly tumorigenic osteosarcoma cell lines (MG-63, Saos-2, and U-2 OS) were analyzed using a publicly accessible web-based platform: Enrichr (http:/ / amp.pharm.mssm.edu/Enrichr/) [27, 28]. Signaling pathway enrichment analysis was performed by Kyoto Encyclopedia of Genes and Genomes (KEGG) pathway analysis, while biological process enrichment analysis was performed by gene ontology (GO) analysis. For PPI network enrichment and gene function analyses, STRING (https://stringdb.org/) was used.

\section{Kaplan-Meier analysis}

Kaplan-Meier survival plots were generated using the R2: Genomics Analysis and Visualization Platform (http://r2.amc.nl). Briefly, osteosarcoma patients were divided into two groups based on the expression value of indicated genes, and the survival rate between the two groups was tested via log-rank test with $P$-value $<0.05$ considered significant. 


\section{Statistical analysis}

All data were analyzed with GraphPad Prism 6 software and are provided as the mean \pm standard error of the mean (SEM) unless otherwise indicated. Statistical analyses were performed using an unpaired Student's t-test. $\mathrm{P}<0.05$ was considered to indicate statistical significance.

\section{Results}

\section{Tumorigenicity assay of human osteosarcoma cell lines}

Six osteosarcoma cell lines (MG-63, Saos-2, U-2 OS, 143B, MNNG/HOS, and SJSA-1) were used for tumorigenicity testing in $\mathrm{BALB} / \mathrm{c}$ nude mice. At 30 days, all five mice formed tumors with 143B, MNNG/HOS, and SJSA-1cells, while none of the five mice formed tumors with MG-63, Saos-2, or U-2 OS cells (Figure 1A). The 143B, MNNG/HOS, and SJSA-1 cell lines formed tumors very rapidly, reaching a volume of $\sim 1,000 \mathrm{~mm}^{3}$ within 30 days. In addition, MNNG/HOS showed the highest tumorigenicity compared with 143B and SJSA-1 (Figure 1B).

\section{GEO and PPI analyses}

According to the tumorigenicity assay, the six osteosarcoma cell lines were divided into the highly tumorigenic group (143B, MNNG/HOS, and SJSA-1) and lowly tumorigenic group (MG-63, Saos-2, and U-2 OS). GEO datasets (GSE36001 and GSE42352) were analyzed with the GEO2R online tool. We set the threshold as $\mathrm{P}$-value $<0.05$ and $\log 2$ fold change (FC) $>1$. Compared with the lowly tumorigenic group, 106 and 106 lowly expressed genes and 177 and 159 highly expressed genes were identified from GSE36001 and GSE42352, respectively, in the highly tumorigenic group (Figure 2A \& B, Table S1). By combining GSE36001 and GSE42352, 86 lowly
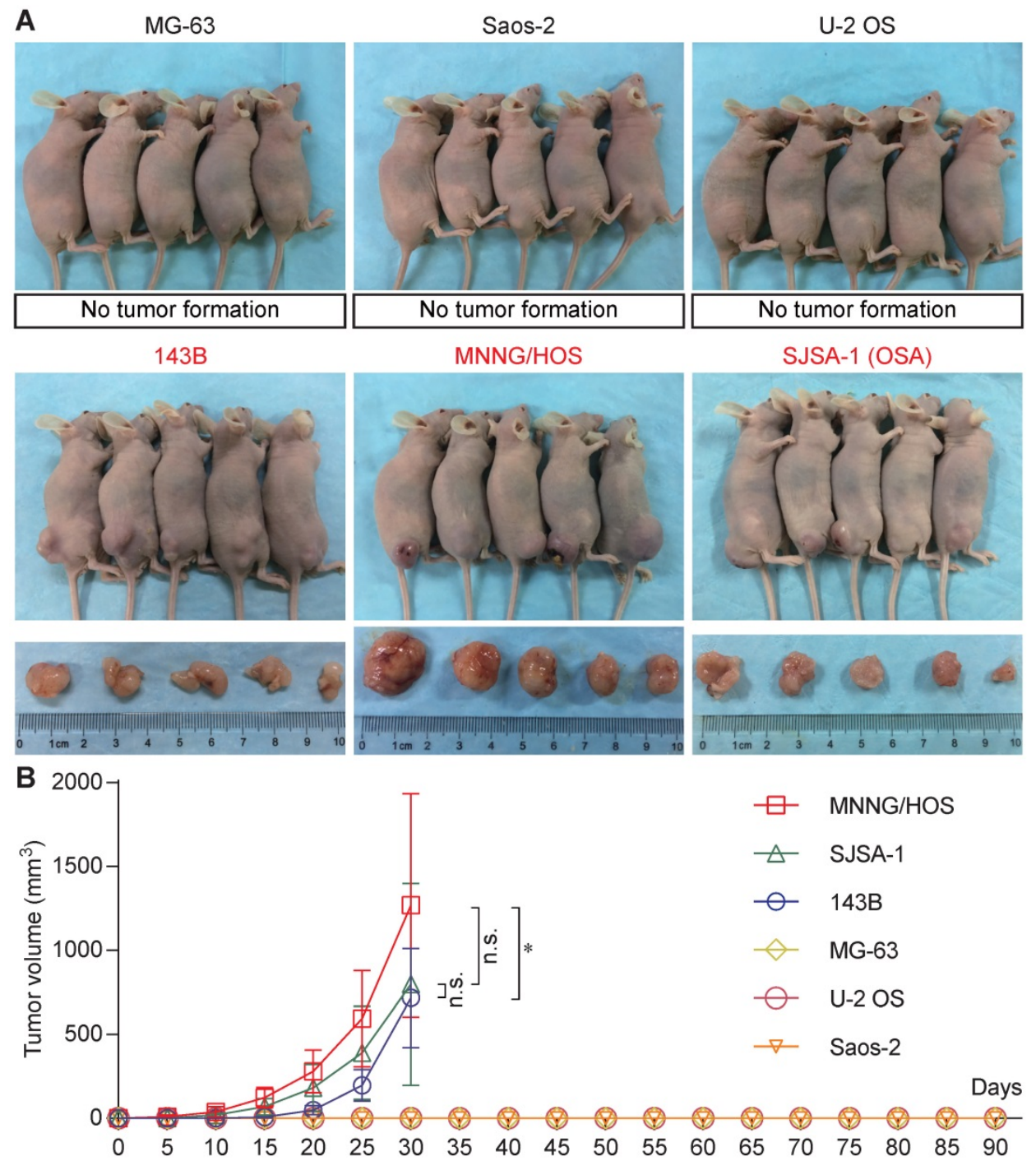

Figure 1. Tumorigenicity assay of human osteosarcoma cell lines. (A) Tumorigenicity of six osteosarcoma cell lines (MG-63, Saos-2, U-2 OS, 143B, MNNG/HOS, and SJSA-1) in vivo. (B) Tumor growth curve based on tumor volume ( ${ }^{\mathrm{P}}<0.05$, n.s.: no significant by two-way ANOVA). 
expressed genes and 138 highly expressed genes were identified (Figure 2C \& D, Table S1). PPI analysis showed that 53 of 86 lowly expressed genes (Figure S1) and 89 of 137 highly expressed genes (Figure S2) were not included in the main PPI network, although the PPI enrichment P-value $=1.67 \mathrm{e}-05$ for lowly expressed genes and 0.00961 for highly expressed genes. The low physical protein-protein interaction of these genes suggested that tumorigenicity involves various biological processes.

\section{Signaling pathway (KEGG) and biological process (GO) enrichment analyses}

Eighty-six lowly expressed genes and 138 highly expressed genes were subjected to further KEGG and GO pathway analysis via Enrichr. The KEGG enrichment analysis revealed that lowly expressed genes were largely correlated with cell cycle, DNA replication and repair, virus infection, and various cancers (Figure 3A), whereas highly expressed genes were correlated with amino acid metabolism and glucose metabolism (Figure 3B). The GO enrichment analysis showed that lowly expressed genes corresponded with positive regulation of extracellular matrix assembly and response to DNA damage
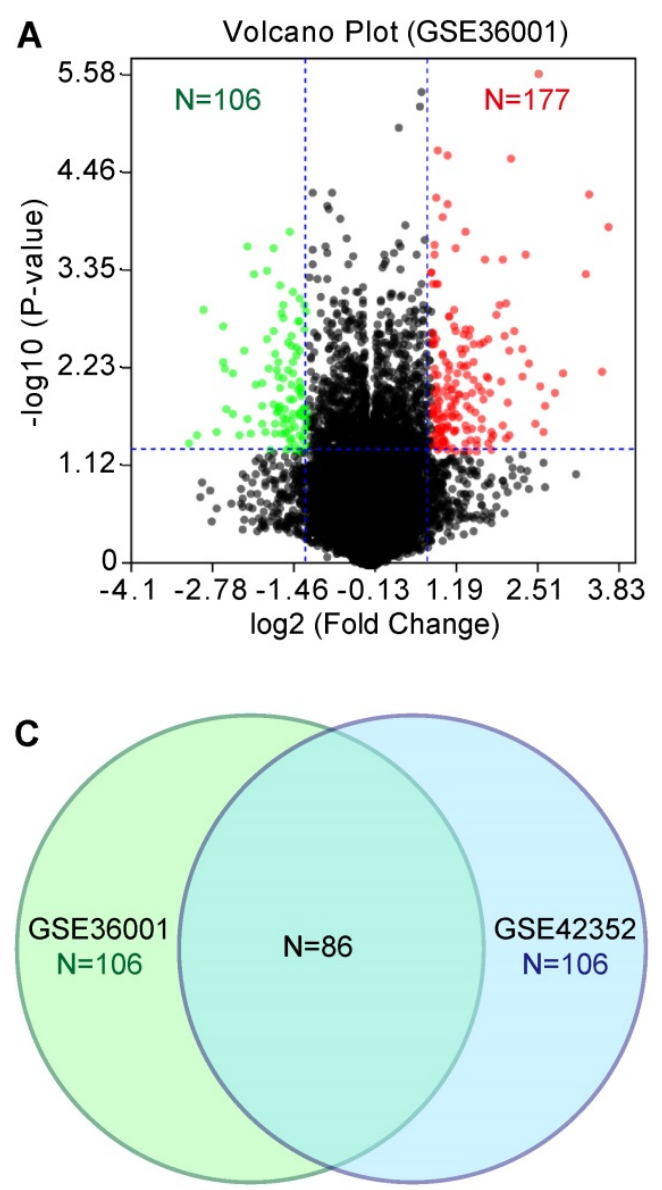

stimulus and negative regulation of mitochondrial outer membrane permeabilization, cell proliferation, and growth (Figure 3C); highly expressed genes corresponded with response to endoplasmic reticulum (ER) stress, aggrephagy, positive regulation of adaptive immune response, and amino sugar metabolic process (Figure 3D).

\section{Identification of candidate lowly expressed and highly expressed genes}

Because of the low relevance of differentially expressed genes, further enrichment analysis was performed. For highly expressed genes, we set the threshold as $\mathrm{P}$-value $<0.01$ and $\log 2 \mathrm{FC}>2$, and for lowly expressed genes, we set the threshold as $P$-value $<0.005$ and $\log 2 \mathrm{FC}>2$. Six lowly expressed genes (CRIP2, CYP26B1, DPYSL4, GP1BB, IFI44, and $P A L L D)$ and 6 highly expressed genes (ARHGDIB, DDX10, DUSP5, FOXA2, HEY1, and TRIB3) were identified (Figure 4A-D). However, the functions of these genes were quite different according to the PPI analysis (PPI enrichment P-value $=1$ ) (Figure 4E \& F). Of note, HEY1 had an extremely low P-value in both GSE36001 and GSE42352.
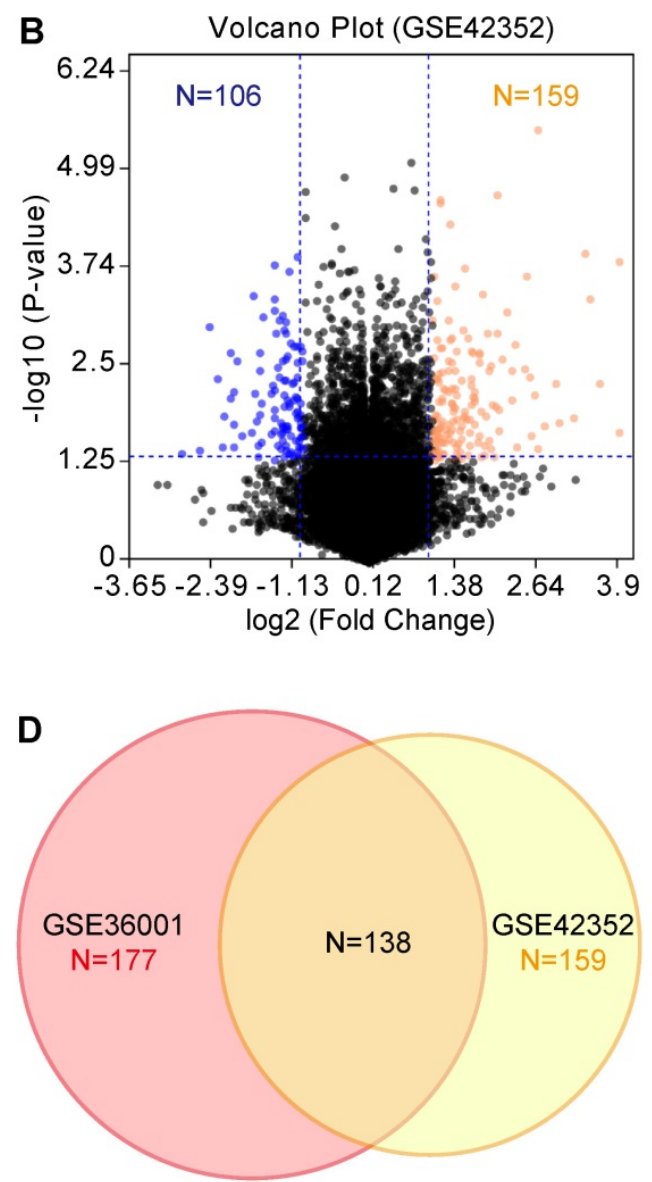

Figure 2. GEO analysis of differentially expressed genes. (A) \& (B) Volcano plot of differentially expressed genes based on GSE36001 and GSE42352, respectively. (C) Differentially lowly expressed genes. (D) Differentially highly expressed genes. 
A KEGG pathway enrichment (combined score)-low expression group

\begin{tabular}{|c|c|}
\hline Cell cycle_Homo sapiens_hsa04110 & $p=1.577 e-5$ \\
\hline DNA replication_Homo sapiens_hsa03030 & $p=4.947 e-4$ \\
\hline HTLV-I infection_Homo sapiens_hsa05166 & $p=5.122 e-3$ \\
\hline Small cell lung cancer_Homo sapiens_hsa05222 & $p=6.076 e-3$ \\
\hline Transcriptional misregulation in cancer_Homo sapiens_hsa05202 & $p=7.598 \mathrm{e}-3$ \\
\hline Bladder cancer_Homo sapiens_hsa05219 & $p=1.344 \mathrm{e}-2$ \\
\hline Base excision repair_Homo sapiens_hsa03410 & $p=8.85 e-3$ \\
\hline Hepatitis B_Homo sapiens_hsa05161 & $\mathrm{p}=2.511 \mathrm{e}-2$ \\
\hline Pathogenic Escherichia coli infection_Homo sapiens_hsa05130 & $p=2.342 e-2$ \\
\hline Chronic myeloid leukemia_Homo sapiens_hsa05220 & $p=3.945-2$ \\
\hline
\end{tabular}

B KEGG pathway enrichment (combined score)-high expression group

\begin{tabular}{|c|c|}
\hline Biosynthesis of amino acids_Homo sapiens_hsa01230 & $p=1.673 e-3$ \\
\hline Cysteine and methionine metabolism_Homo sapiens_hsa00270 & $p=3.616 e-3$ \\
\hline Insulin resistance_Homo sapiens_hsa04931 & $p=6.733 e-3$ \\
\hline Apoptosis_Homo sapiens_hsa04210 & $\mathrm{p}=1.579 \mathrm{e}-2$ \\
\hline 2-Oxocarboxylic acid metabolism_Homo sapiens_hsa01210 & $p=5.922 e-3$ \\
\hline MAPK signaling pathway_Homo sapiens_hsa04010 & $p=3.119 e-2$ \\
\hline Fc gamma R-mediated phagocytosis_Homo sapiens_hsa04666 & $p=2.609 e-2$ \\
\hline SNARE interactions in vesicular transport_Homo sapiens_hsa04130 & $p=2.265 e-2$ \\
\hline \multicolumn{2}{|c|}{ Alanine, aspartate and glutamate metabolism_Homo sapiens_hsa00250 p=2.391e-2 } \\
\hline Glycine, serine and threonine metabolism_Homo sapiens_hsa00260 & $p=3.066 e-2$ \\
\hline $\begin{array}{lr}4 & 6 \\
\text { combined score }\end{array}$ & 10 \\
\hline
\end{tabular}

C GO Biological Process enrichment (combined score)-low expression group

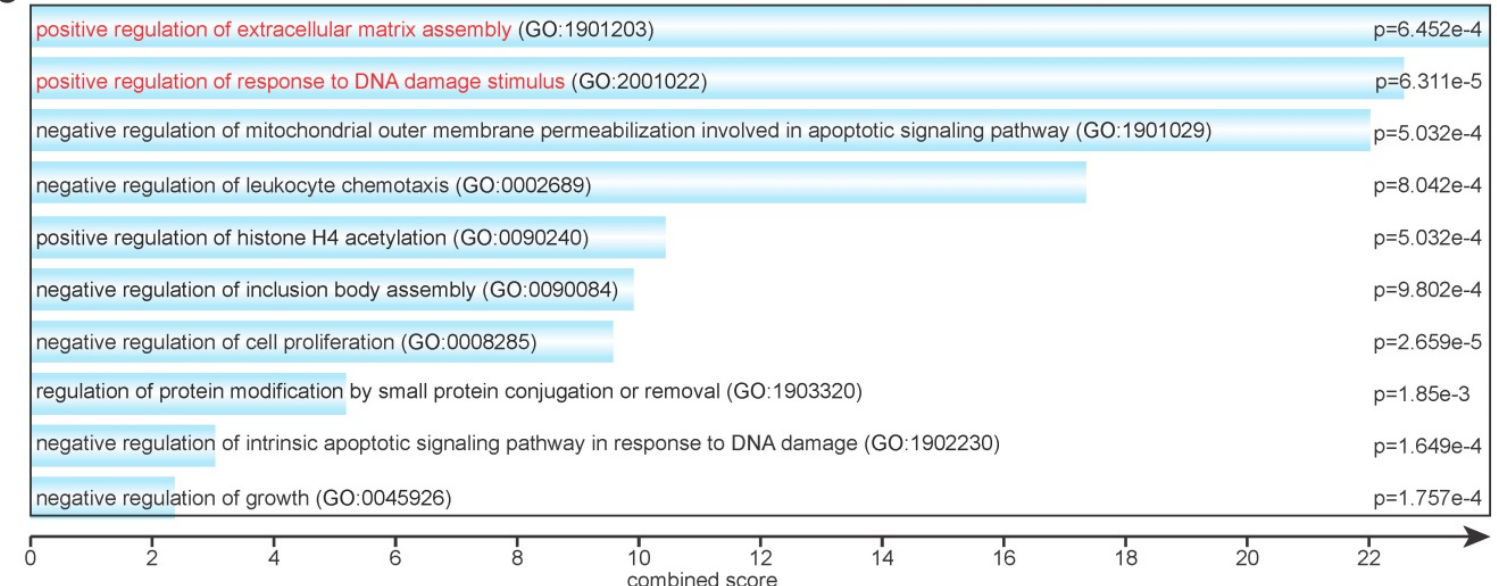

D

GO Biological Process enrichment (combined score)-high expression group

\begin{tabular}{|c|c|}
\hline intrinsic apoptotic signaling pathway in response to endoplasmic reticulum stress (GO:0070059) & $p=2.384 e-5$ \\
\hline aggrephagy (GO:0035973) & $p=9.564 e-4$ \\
\hline positive regulation of adaptive immune response (GO:0002821) & $p=9.564 e-4$ \\
\hline amino sugar metabolic process (GO:0006040) & $p=2.46 e-3$ \\
\hline regulation of intracellular signal transduction (GO:1902531) & $p=1.62 e-4$ \\
\hline negative regulation of endoplasmic reticulum unfolded protein response (GO:1900102) & $p=2.46 e-3$ \\
\hline pri-miRNA transcription from RNA polymerase II promoter (GO:0061614) & $p=1.27 e-3$ \\
\hline activation of cysteine-type endopeptidase activity (GO:0097202) & $p=3.458 \mathrm{e}-3$ \\
\hline apoptotic process (GO:0006915) & $p=1.109 e-3$ \\
\hline negative regulation of peptidyl-threonine phosphorylation (GO:0010801) & $p=5.246 e-3$ \\
\hline
\end{tabular}

Figure 3. Signaling pathway (KEGG) and biological process (GO) enrichment analyses. (A) KEGG analysis of differentially lowly expressed genes. (B) KEGG analysis of differentially highly expressed genes. (C) GO analysis of differentially lowly expressed genes. (D) GO analysis of differentially highly expressed genes.

\section{Prognostic analysis and $\mathrm{qPCR}$ validation}

Based on the candidate lowly expressed and highly expressed genes, the R2 Genomics Platform was used for prognostic analysis. High expression of CYP26B1, GP1BB, and IFI44 in osteosarcoma patients was correlated with good prognosis (Figure 5A-C), while high expression of DDX10 and FOXA2 in osteosarcoma patients was associated with poor prognosis (Figure 5D \& E). Notably, high expression of HEY1 corresponded with poor prognosis (both overall survival and metastasis-free survival) (Figure $5 F \& G)$. qPCR confirmed that the expression changes were consistent with the microarray datasets (Figure $5 \mathrm{H})$. 


\section{Importance of HEY 1 in the tumorigenicity of 143B osteosarcoma cells}

The protein expression level of HEY1 detected by western blot was consistent with the mRNA level in osteosarcoma cell lines (Figure 6A). Western blot analysis of changes in HEY1 expression showed that shHEY1-2 is more effective (Figure 6B) under an equivalent infection rate (Figure 6C). The 143B osteosarcoma cells with red fluorescent protein (mStrawberry) were visualized under visible light (Figure 6D) to monitor cell growth (Figure 6E). The appearance of subcutaneous tumors at the experimental endpoint (Figure 6E \& F), tumor growth curves (Figure 6G), and tumor weight (Figure 6H) showed that knockdown of HEY1 mRNA inhibited the tumorigenicity significantly. On the other hand, overexpression of HEY1 in a non-tumorigenic cell line Saos-2 (Figure 6I) could help Saos-2 cells to obtain the tumorigenicity (Figure 6J-M). These results revealed that HEY1 is a key factor in the tumorigenicity of $143 \mathrm{~B}$ osteosarcoma cells.

\section{Discussion}

Identifying the tumorigenicity-associated genes in osteosarcoma is a valuable method for investigation of tumorigenic mechanisms. In this study, we evaluated the tumor-forming ability of six osteosarcoma cell lines. Three (143B, MNNG/HOS, and SJSA-1) were found to be tumorigenic, while the other three cell lines (MG-63, Saos-2, and U-2 OS) lacked tumorigenicity (Figure 1A). Thus, 143B, MNNG/HOS, and SJSA-1 were defined as the highly tumorigenic group, while MG-63, Saos-2, and U-2 OS were defined as the poorly tumorigenic group. Two microarray datasets from two independent studies containing the mRNA expression data of these six cell lines were used for analysis of differentially expressed genes.
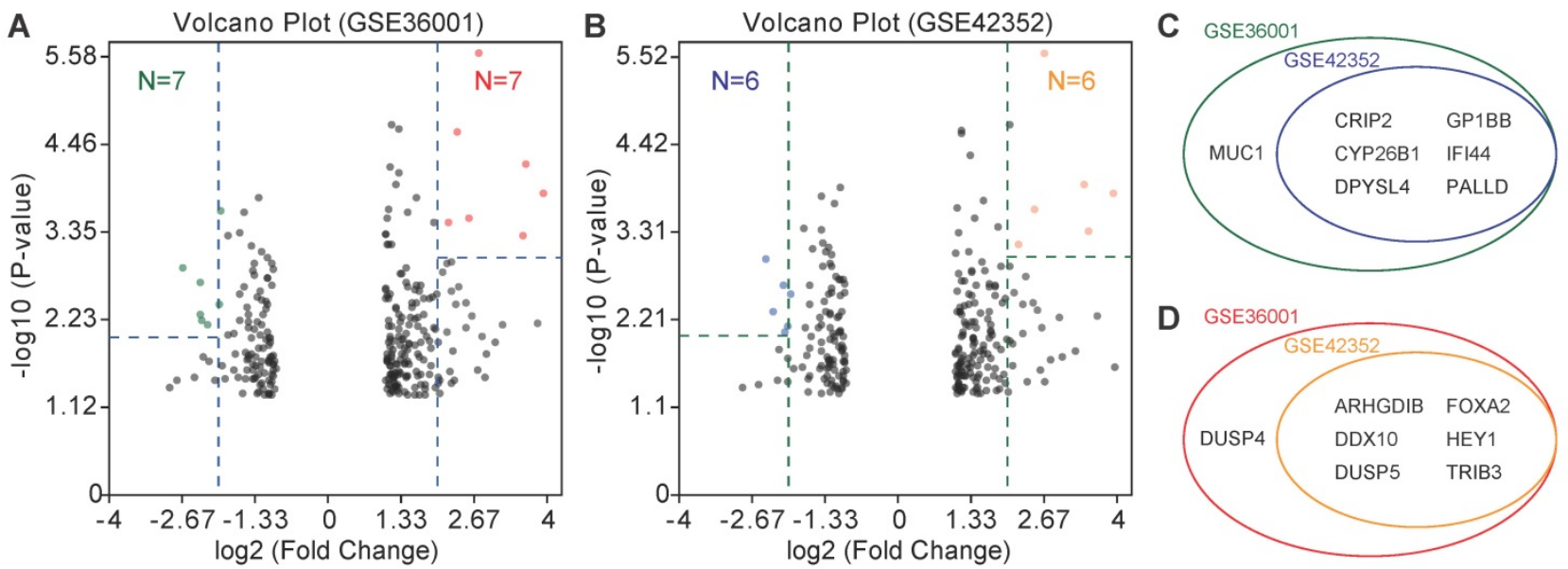

E

Low-expression genes in tumorigenic osteosarcoma cell lines

\begin{tabular}{|c|c|c|c|c|c|c|c|c|c|c|}
\hline \multirow[t]{2}{*}{ Gene } & \multicolumn{2}{|c|}{ GSE36001 } & \multicolumn{2}{|c|}{ GSE42352 } & \multicolumn{2}{|l|}{ KEGG Pathways } & \multicolumn{2}{|c|}{ Biological Process (GO) } & \multicolumn{2}{|c|}{ Molecular Function (GO) } \\
\hline & $P$ value & Log2 FC & $P$ value & Log2 FC & pathway description & FDR & pathway description & FDR & pathway description & FDR \\
\hline CRIP2 & $5.59 e-3$ & -2.366 & $8.16 e-3$ & -2.129 & cGMP-PKG pathway & $2.01 \mathrm{e}-2$ & organ development & $2.65 \mathrm{e}-3$ & transcriptional activity & $5.08 e-4$ \\
\hline CYP26B1 & $1.84 \mathrm{e}-3$ & -2.395 & $2.13 e-3$ & -2.139 & retinol metabolism & $4.66 \mathrm{e}-23$ & xenobiotic metabolism & $1.81 \mathrm{e}-11$ & oxidoreductase activity & $5.39 e-15$ \\
\hline DPYSL4 & $1.2 \mathrm{e}-3$ & -2.701 & $9.85 e-4$ & -2.458 & axon guidance & $9.33 e-7$ & pyrimidine biosynthesis & $1.06 e-5$ & carbon-nitrogen ligase & $1.04 e-4$ \\
\hline GP1BB & $4.69 e-3$ & -2.384 & $4.47 e-3$ & -2.329 & complement/coagulation & $4.56 \mathrm{e}-8$ & blood coagulation & $2.13 e-23$ & $\longrightarrow$ & - \\
\hline IFI44 & $6.38 \mathrm{e}-3$ & -2.244 & $6.85 \mathrm{e}-3$ & -2.065 & influenza A & $2.37 e-2$ & response to virus & $2.53 e-15$ & - & - \\
\hline PALLD & $3.38 \mathrm{e}-3$ & -2.039 & $2.73 e-3$ & -2.021 & cytoskeleton regulation & $4.62 \mathrm{e}-2$ & actin filament assembly & $9.05 e-3$ & actin binding & $6.13 e-3$ \\
\hline
\end{tabular}

\begin{tabular}{|c|c|c|c|c|c|c|c|c|c|c|}
\hline \multirow{2}{*}{ Gene } & \multicolumn{2}{|c|}{ GSE36001 } & \multicolumn{2}{|c|}{ GSE42352 } & \multicolumn{2}{|l|}{ KEGG Pathways } & \multicolumn{2}{|c|}{ Biological Process (GO) } & \multicolumn{2}{|c|}{ Molecular Function (GO) } \\
\hline & $\underline{P \text { value }}$ & Log2 FC & $\underline{P \text { value }}$ & Log2 FC & pathway description & FDR & pathway description & FDR & pathway description & FDR \\
\hline ARHGDIB & $5.72 e-5$ & 3.566 & $1.12 \mathrm{e}-4$ & 3.348 & axon guidance & $5.4 e-9$ & small GTPase regulation & $1.34 \mathrm{e}-18$ & GTP binding & $1.19 e-13$ \\
\hline DDX10 & $3.14 \mathrm{e}-4$ & 2.154 & $6.39 e-4$ & 2.156 & ribosome biogenesis & $2.02 \mathrm{e}-3$ & ribosome biogenesis & $3.31 e-4$ & poly(A) RNA binding & $5.1 \mathrm{e}-11$ \\
\hline DUSP5 & $4.65 e-4$ & 3.509 & $4.38 \mathrm{e}-4$ & 3.439 & MAPK pathway & $2.19 \mathrm{e}-19$ & TLR pathway & $3.72 \mathrm{e}-12$ & MAP kinase activity & $1.15 \mathrm{e}-21$ \\
\hline FOXA2 & $2.69 e-4$ & 2.52 & $2.28 e-4$ & $2.444 \mathrm{ma}$ & urity onset diabetes (young & $1.63 e-7$ & insulin secretion regulatio & on $1.17 \mathrm{e}-8$ & transcriptional activity & $3.07 e-6$ \\
\hline HEY1 & $2.61 e-6$ & 2.898 & $3.03 e-6$ & 2.634 & Notch signaling pathway & $6.75 e-9$ st & triated muscle developmen & nt $6.71 \mathrm{e}-8$ & transcriptional activity & $1.25 e-7$ \\
\hline TRIB3 & $1.35 \mathrm{e}-4$ & 3.871 & $1.46 \mathrm{e}-4$ & 3.892 & prostate cancer & $5.61 \mathrm{e}-10$ & response to insulin & $2.29 \mathrm{e}-5$ & transcriptional activity & $3.08 \mathrm{e}-4$ \\
\hline
\end{tabular}

Figure 4. Identification of candidate lowly expressed and highly expressed genes. (A) \& (B) Volcano plot of candidate lowly expressed and highly expressed genes. (C) \& (D) Lowly and highly expressed genes. e Function enrichment of lowly expressed genes. f Function enrichment of highly expressed genes. Log2 FC: Log2 fold change; FDR: False discovery rate. 

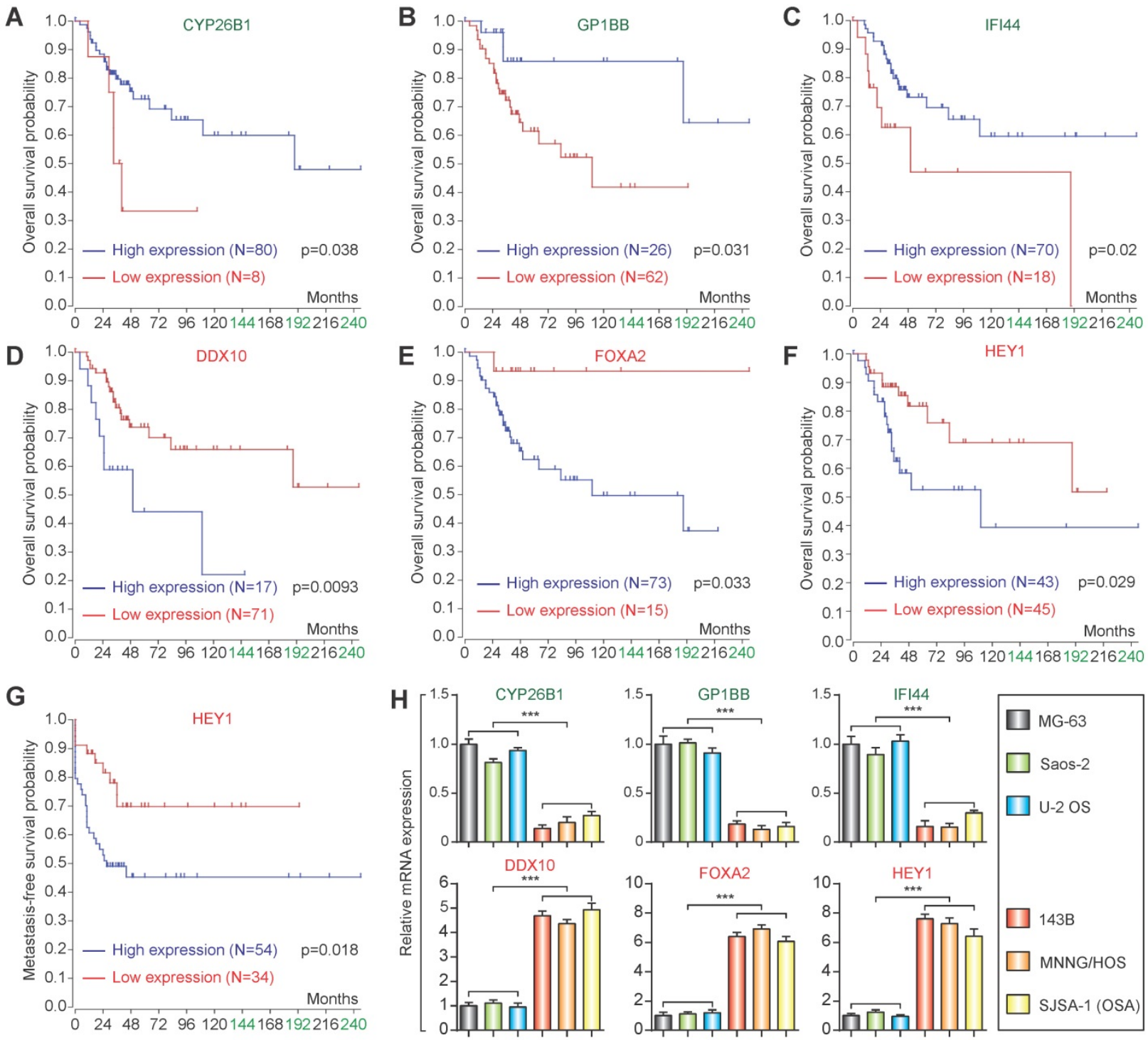

Figure 5. Prognostic analysis and qPCR validation. Overall survival analysis of CYP26BI (A), GPIBB (B), IFI44 (C), DDXIO (D), FOXA2 (E) and HEYI (F). (G) Metastasis-free survival of HEYI. (H) qPCR validation of identification of candidate lowly expressed and highly expressed genes. (***P $<0.001$ by student's $t$-test).

It is generally accepted that the genes that promote cell cycle progression and DNA replication act as positive factors in tumorigenesis [29]. However, tumorigenicity is not always correlated with tumorigenesis or proliferation. The poorly tumorigenic MG-63 and U-2 OS cells showed the same proliferation rate as the highly tumorigenic 143B and SJSA-1 cells [24]. KEGG analysis showed that the cell cycle- and DNA replication-related genes were lowly expressed in the highly tumorigenic group, while amino acid metabolism- and glucose metabolismrelated genes were highly expressed in the highly tumorigenic group (Figure $3 \mathrm{~A} \& \mathrm{~B}$ ). Thus, we speculate that amino acid- and energy metabolismassociated genes are important to the tumorigenicity. In contrast, vigorous metabolism may help the osteosarcoma cells adapt to the environmental alteration [30]. Coincidentally, GO analysis showed that the intrinsic apoptosis- and aggrephagy-related genes were highly expressed in the highly tumorigenic group. It may help osteosarcoma cells overcome the temporary nutrition deficiency and adapt to the bioenergetic challenges in vivo [31, 32].

PPI analysis showed low relevance among the differentially expressed genes, which suggests that tumorigenicity is a complicated process involving various genes and biological pathways. Additional enrichment analysis identified six lowly expressed and six highly expressed genes. Nonetheless, there was no relevance among these differentially expressed genes. Half of these differentially expressed genes were correlated with good or poor prognosis. Indeed, one of the shortcomings of prognosis analysis for rare pediatric tumors, such as this study, is the lack of adequate samples to power the discovery of rare events. 

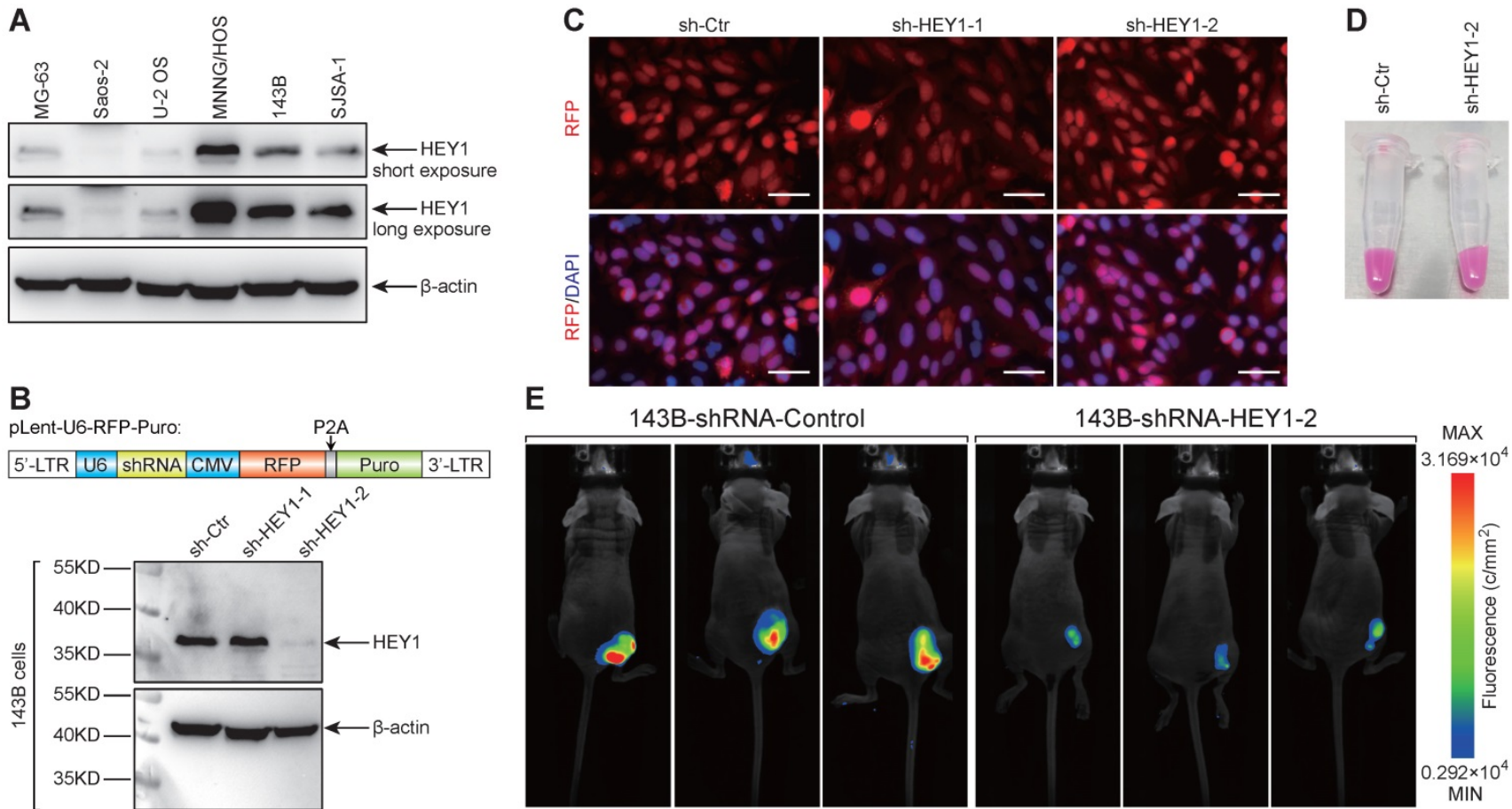

B
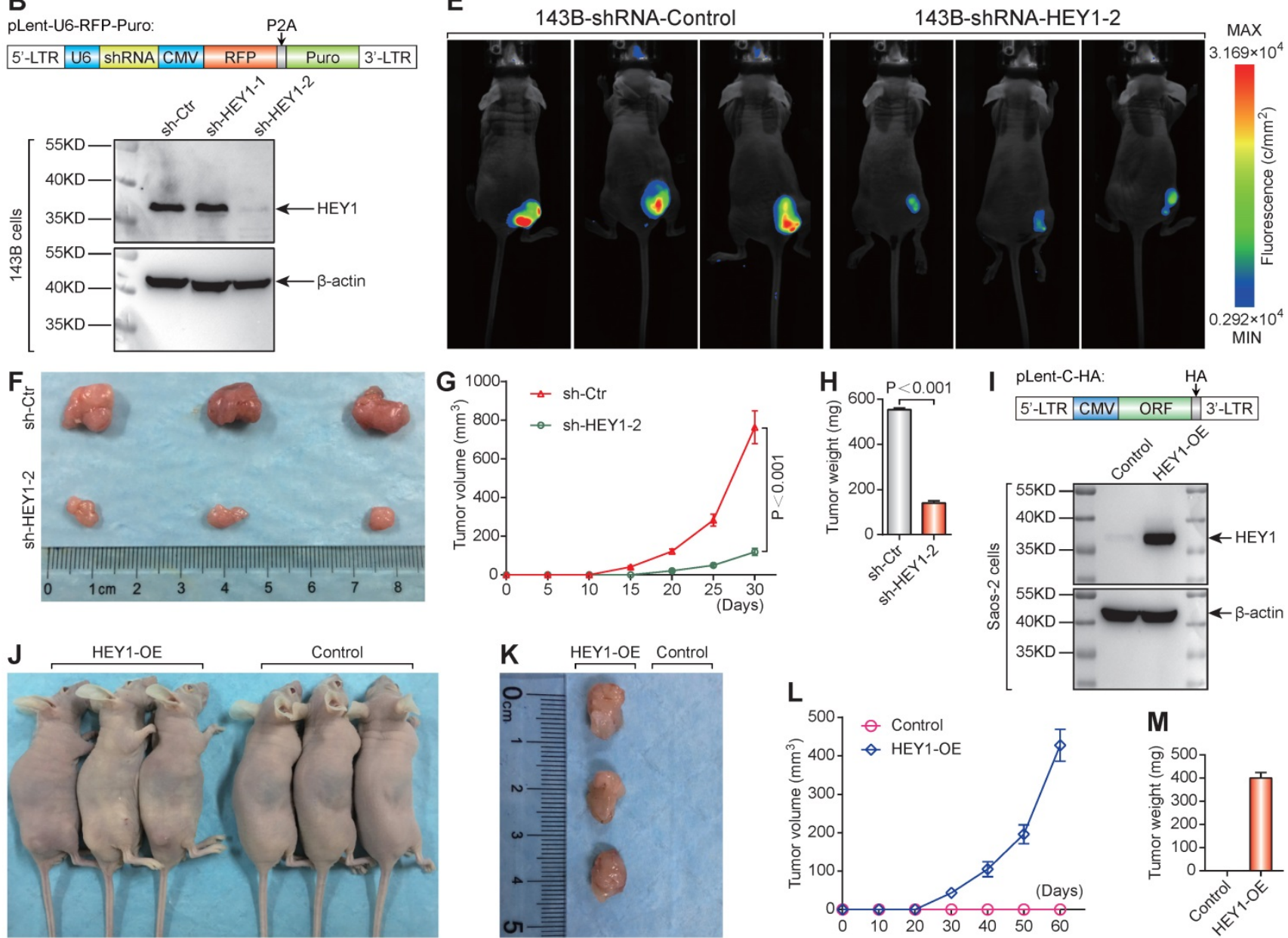

Figure 6. HEY1 is a key factor in the tumorigenicity of 143B osteosarcoma cells. (A) Protein expression level of HEY1 in six osteosarcoma cell lines (MG-63, Saos-2, U-2 OS, MNNG/HOS, 143B, and SJSA-1). (B) Protein expression level of HEY1 after lentivirus-mediated RNA interference in 143B osteosarcoma cells. (C) Lentivirus infection efficiency detected by fluorescence microscopy (scale bar $=200 \mu \mathrm{m}$ ). (D) Lentivirus-infected 143B osteosarcoma cell pellet under visible light. (E) Images of 143B cell-bearing mice under 530-nm laser irradiation at the experimental endpoint. Subcutaneous tumors at the experimental endpoint (F), tumor growth curves (G), and tumor weight (H) are shown. (I) Protein expression level of HEY1 in Saos-2 and HEY1-overexpressed Saos-2 cells. (J) Tumorigenicity assay of Saos-2 cells after HEY1-overexpression. Subcutaneous tumors at the experimental endpoint (K), tumor growth curves (L), and tumor weight (M) of Saos-2 cells after HEY1-overexpression are shown.

We noticed that HEY1 was highly expressed in the highly tumorigenic group with an extremely low P-value in both GSE36001 and GSE42352. In addition, the expression level of $H E Y 1$ was positively correlated with the tumorigenicity of osteosarcoma cell lines. Thus, HEY1 was selected for further investigation. In fact, HEY1 is a candidate oncogene that is highly expressed in glioblastoma [33, 34], rhabdomyosarcoma [35], hepatocellular carcinoma [36], head and neck squamous cell carcinoma [37], and renal cell carcinoma [38], among other cancers. It is also associated with poor prognosis in head and neck squamous cell carcinoma [37] and esophageal squamous cell carcinoma [39]. On the other hand, HEY1 acts a target gene of Delta-like canonical Notch ligand 4 (Dll4)-induced Notch signaling pathway activation and functions as an inhibitor of myogenesis [40]. Our data also proved that HEY1 is a key factor 
involved in the tumorigenicity of osteosarcoma cells. We anticipate that this result will promote preclinical studies on this malignant tumor.

In summary, amino acid- and energy metabolism-associated genes are important to the tumorigenicity of osteosarcoma cells. Limited cell cycle progression and high level of amino acids and energy metabolism as well as aggrephagy may help osteosarcoma cells overcome the temporary nutrition deficiency and survive in vivo. Furthermore, HEY1 is a key factor in the tumorigenicity of osteosarcoma cells. These results provide not only a new perspective to understand the mechanism of tumorigenicity but also another strategy for preclinical studies and targeted therapy of this orphan disease.

\section{Supplementary Material}

Supplementary figures and table legend.

http://www.jcancer.org/v11p3623s1.pdf

Supplementary table.

http://www.jcancer.org/v11p3623s2.xlsx

\section{Acknowledgements}

We thank Professor Leonardo A. Meza-Zepeda (Oslo University, Norway) and Professor Anne-Marie Cleton-Jansen (Leiden University, the Netherlands) who provided extremely reliable microarray data (GSE36001 and GSE42352, respectively).

\section{Funding}

The National Key Research and Development Program of China 2016YFA0100900 to JS; General Program of National Natural Science Foundation of China 81871403 to JS; The Zhejiang Provincial Key Research and Development Program of China 2019C03014 to J.S.; Zhejiang Provincial Natural Science Foundation of China LY20H160035 to MZ, LY15H030011 to LZ, LY19H030013 to RM, \& GF18H180028 to XX; General Program of National Natural Science Foundation of China 61976075 to XX; Medical and Health Program of Zhejiang Province 2017209189 (2017KY383) to MZ, 2019338991 to FZ \& 2016KYB078 to JZ.

\section{Competing Interests}

The authors have declared that no competing interest exists.

\section{References}

1. Isakoff MS, Bielack SS, Meltzer P, Gorlick R. Osteosarcoma: Current Treatment and a Collaborative Pathway to Success. J Clin Oncol. 2015; 33: 3029-35.

2. Kansara M, Teng MW, Smyth MJ, Thomas DM. Translational biology of osteosarcoma. Nat Rev Cancer. 2014; 14: 722-35.

3. Link MP, Goorin AM, Miser AW, Green AA, Pratt CB, Belasco JB, et al. The effect of adjuvant chemotherapy on relapse-free survival in patients with osteosarcoma of the extremity. N Engl J Med. 1986; 314: 1600-6.

4. Ferrari S, Smeland S, Mercuri M, Bertoni F, Longhi A, Ruggieri P, et al. Neoadjuvant chemotherapy with high-dose Ifosfamide, high-dose methotrexate, cisplatin, and doxorubicin for patients with localized osteosarcoma of the extremity: a joint study by the Italian and Scandinavian Sarcoma Groups. J Clin Oncol. 2005; 23: 8845-52.

5. Bielack SS, Kempf-Bielack B, Delling G, Exner GU, Flege S, Helmke K, et al. Prognostic factors in high-grade osteosarcoma of the extremities or trunk: an analysis of 1,702 patients treated on neoadjuvant cooperative osteosarcoma study group protocols. J Clin Oncol. 2002; 20: 776-90.

6. Hogendoorn PC, Group EEW, Athanasou N, Bielack S, De Alava E, Dei Tos $\mathrm{AP}$, et al. Bone sarcomas: ESMO Clinical Practice Guidelines for diagnosis, treatment and follow-up. Ann Oncol. 2010; 21 Suppl 5: v204-13.

7. Collins M, Wilhelm M, Conyers R, Herschtal A, Whelan J, Bielack S, et al. Benefits and adverse events in younger versus older patients receiving neoadjuvant chemotherapy for osteosarcoma: findings from a meta-analysis. J Clin Oncol. 2013; 31: 2303-12.

8. Meyers PA, Healey JH, Chou AJ, Wexler LH, Merola PR, Morris CD, et al. Addition of pamidronate to chemotherapy for the treatment of osteosarcoma. Cancer. 2011; 117: 1736-44.

9. Yang J, Zhao L, Tian W, Liao Z, Zheng H, Wang G, et al. Correlation of WWOX, RUNX2 and VEGFA protein expression in human osteosarcoma. BMC Med Genomics. 2013; 6: 56.

10. Yang J, Yang D, Sun Y, Sun B, Wang G, Trent JC, et al. Genetic amplification of the vascular endothelial growth factor (VEGF) pathway genes, including VEGFA, in human osteosarcoma. Cancer. 2011; 117: 4925-38.

11. Lonardo F, Ueda T, Huvos AG, Healey J, Ladanyi M. p53 and MDM2 alterations in osteosarcomas: correlation with clinicopathologic features and proliferative rate. Cancer. 1997; 79: 1541-7.

12. Yotov WV, Hamel H, Rivard GE, Champagne MA, Russo PA, Leclerc JM, et al. Amplifications of DNA primase 1 (PRIM1) in human osteosarcoma. Genes Chromosomes Cancer. 1999; 26: 62-9.

13. Wunder JS, Gokgoz N, Parkes R, Bull SB, Eskandarian S, Davis AM, et al. TP53 mutations and outcome in osteosarcoma: a prospective, multicenter study. J Clin Oncol. 2005; 23: 1483-90.

14. Toguchida J, Ishizaki K, Sasaki MS, Nakamura Y, Ikenaga M, Kato M, et al. Preferential mutation of paternally derived RB gene as the initial event in sporadic osteosarcoma. Nature. 1989; 338: 156-8

15. Freeman SS, Allen SW, Ganti R, Wu J, Ma J, Su X, et al. Copy number gains in EGFR and copy number losses in PTEN are common events in osteosarcoma tumors. Cancer. 2008; 113: 1453-61.

16. Berger M, Grignani G, Giostra A, Ferrari S, Ferraresi V, Tamburini A, et al. 153Samarium-EDTMP administration followed by hematopoietic stem cell support for bone metastases in osteosarcoma patients. Ann Oncol. 2012; 23: 1899-905.

17. Grignani G, Palmerini E, Dileo P, Asaftei SD, D'Ambrosio L, Pignochino Y, et al. A phase II trial of sorafenib in relapsed and unresectable high-grade osteosarcoma after failure of standard multimodal therapy: an Italian Sarcoma Group study. Ann Oncol. 2012; 23: 508-16.

18. Ebb D, Meyers P, Grier H, Bernstein M, Gorlick R, Lipshultz SE, et al. Phase II trial of trastuzumab in combination with cytotoxic chemotherapy for treatment of metastatic osteosarcoma with human epidermal growth factor receptor 2 overexpression: a report from the children's oncology group. J Clin Oncol. 2012; 30: 2545-51.

19. Weigel B, Malempati S, Reid JM, Voss SD, Cho SY, Chen HX, et al. Phase 2 trial of cixutumumab in children, adolescents, and young adults with refractory solid tumors: a report from the Children's Oncology Group. Pediatr Blood Cancer. 2014; 61: 452-6.

20. Chawla SP, Staddon AP, Baker LH, Schuetze SM, Tolcher AW, D'Amato GZ, et al. Phase II study of the mammalian target of rapamycin inhibitor ridaforolimus in patients with advanced bone and soft tissue sarcomas. J Clin Oncol. 2012; 30: 78-84.

21. Arndt CA, Koshkina NV, Inwards CY, Hawkins DS, Krailo MD, Villaluna D, et al. Inhaled granulocyte-macrophage colony stimulating factor for first pulmonary recurrence of osteosarcoma: effects on disease-free survival and immunomodulation. a report from the Children's Oncology Group. Clin Cancer Res. 2010; 16: 4024-30.

22. Anderson PM, Meyers P, Kleinerman E, Venkatakrishnan K, Hughes DP, Herzog C, et al. Mifamurtide in metastatic and recurrent osteosarcoma: a patient access study with pharmacokinetic, pharmacodynamic, and safety assessments. Pediatr Blood Cancer. 2014; 61: 238-44.

23. Chou AJ, Kleinerman ES, Krailo MD, Chen Z, Betcher DL, Healey JH, et al. Addition of muramyl tripeptide to chemotherapy for patients with newly diagnosed metastatic osteosarcoma: a report from the Children's Oncology Group. Cancer. 2009; 115: 5339-48.

24. Lauvrak SU, Munthe E, Kresse SH, Stratford EW, Namlos HM, Meza-Zepeda LA, et al. Functional characterisation of osteosarcoma cell lines and identification of mRNAs and miRNAs associated with aggressive cancer phenotypes. Br J Cancer. 2013; 109: 2228-36.

25. Kresse SH, Rydbeck H, Skarn M, Namlos HM, Barragan-Polania AH, Cleton-Jansen AM, et al. Integrative analysis reveals relationships of genetic and epigenetic alterations in osteosarcoma. PLoS One. 2012; 7: e48262.

26. Kuijjer ML, Peterse EF, van den Akker BE, Briaire-de Bruijn IH, Serra M, Meza-Zepeda LA, et al. IR/IGF1R signaling as potential target for treatment of high-grade osteosarcoma. BMC Cancer. 2013; 13: 245.

27. Chen EY, Tan CM, Kou Y, Duan Q, Wang Z, Meirelles GV, et al. Enrichr: interactive and collaborative HTML5 gene list enrichment analysis tool. BMC Bioinformatics. 2013; 14: 128 . 
28. Kuleshov MV, Jones MR, Rouillard AD, Fernandez NF, Duan Q, Wang Z, et al. Enrichr: a comprehensive gene set enrichment analysis web server 2016 update. Nucleic Acids Res. 2016; 44: W90-7.

29. Zhang BN, Bueno Venegas A, Hickson ID, Chu WK. DNA replication stress and its impact on chromosome segregation and tumorigenesis. Semin Cancer Biol. 2018; 55:61-69.

30. Cheong $\mathrm{H}, \mathrm{Lu} \mathrm{C}$, Lindsten $\mathrm{T}$, Thompson CB. Therapeutic targets in cancer cell metabolism and autophagy. Nat Biotechnol. 2012; 30: 671-8.

31. Jiang S, Zhang M, Sun J, Yang X. Casein kinase 1alpha: biological mechanisms and theranostic potential. Cell Commun Signal. 2018; 16: 23.

32. Ichim G, Tait SW. A fate worse than death: apoptosis as an oncogenic process. Nat Rev Cancer. 2016; 16: 539-48.

33. Hulleman E, Quarto M, Vernell R, Masserdotti G, Colli E, Kros JM, et al. A role for the transcription factor HEY1 in glioblastoma. J Cell Mol Med. 2009; 13: 136-46.

34. Tsung AJ, Guda MR, Asuthkar S, Labak CM, Purvis IJ, Lu Y, et al. Methylation regulates HEY1 expression in glioblastoma. Oncotarget. 2017; 8: 44398-409.

35. Roma J, Masia A, Reventos J, Sanchez de Toledo J, Gallego S. Notch pathway inhibition significantly reduces rhabdomyosarcoma invasiveness and mobility in vitro. Clin Cancer Res. 2011; 17: 505-13.

36. Jia D, Wei L, Guo W, Zha R, Bao M, Chen Z, et al. Genome-wide copy number analyses identified novel cancer genes in hepatocellular carcinoma. Hepatology. 2011; 54: 1227-36.

37. Rettig EM, Bishop JA, Agrawal N, Chung $\mathrm{CH}$, Sharma $\mathrm{R}$, Zamuner $\mathrm{F}$, et al. HEY1 is expressed independent of NOTCH1 and is associated with poor prognosis in head and neck squamous cell carcinoma. Oral Oncol. 2018; 82: 168-75.

38. Karim S, Al-Maghrabi JA, Farsi HM, Al-Sayyad AJ, Schulten HJ, Buhmeida A, et al. Cyclin D1 as a therapeutic target of renal cell carcinoma- a combined transcriptomics, tissue microarray and molecular docking study from the Kingdom of Saudi Arabia. BMC Cancer. 2016; 16: 741.

39. Forghanifard MM, Taleb S, Abbaszadegan MR. Notch Signaling Target Genes are Directly Correlated to Esophageal Squamous Cell Carcinoma Tumorigenesis. Pathol Oncol Res. 2015; 21: 463-7.

40. Nandagopal N, Santat LA, LeBon L, Sprinzak D, Bronner ME, Elowitz MB. Dynamic Ligand Discrimination in the Notch Signaling Pathway. Cell. 2018; 172: 869-80 e19. 